\title{
Limbic Encephalitis as a Heralding Manifestation of Primary Sjogren's Syndrome
}

\author{
Rajesh Verma ${ }^{1} \quad$ Rohit Anand ${ }^{1}$ \\ ${ }^{1}$ Department of Neurology, King George's Medical University, \\ Lucknow, Uttar Pradesh, India
}

J Neurosci Rural Pract 2020;11:658-660

\begin{abstract}
Address for correspondence Rajesh Verma, DM, DNB, Department of Neurology, King George's Medical University, Lucknow, Uttar Pradesh 226003, India (e-mail: drrajeshverma32@yahoo.com).
\end{abstract}

\begin{abstract}
Keywords

- primary Sjogren's syndrome

- limbic encephalitis

- autoimmune disorder

- cognitive decline

Sjogren's syndrome (SS) is an autoimmune disorder characterized by lymphocytic and plasma cell infiltration of exocrine glands, resulting in dry mouth and keratoconjunctivitis sicca. The clinical symptoms may range from mucosal dryness to more systemic complaints. We report a 42 -year-old man presenting with rapid cognitive decline and memory impairment for 2 months. Magnetic resonance imaging of the cranium revealed abnormal signals at the bilateral medial temporal lobe and hippocampal region. The autoimmune panel and paraneoplastic antibodies were negative. The patient was labeled as a case of primary SS based on the vasculitis profile and pathological examination of lip biopsy. This case illustrates a rare, heralding manifestation of SS as limbic encephalitis.
\end{abstract}

\section{Introduction}

Primary Sjogren's syndrome(pSS) is an autoimmune disorder with a prevalence of approximately 2 to $3 \%$ in the adult population. SS is characterized by mononuclear cell infiltration and destruction of salivary and lacrimal glands, resulting in dry mouth and keratoconjunctivitis sicca (dry eyes). ${ }^{1}$ The clinical manifestations of pSS are of a broad spectrum, ranging from mucosal dryness to more systemic complaints. Among the neurological presentations, the involvement of the peripheral nervous system is seen more commonly with a frequency of around 10 to $20 \%^{2,3}$ The central nervous system (CNS) involvement is not well established, with a rate varying from 1.5 to $25 \% .{ }^{4,5}$ The CNS manifestations can be in a diverse spectrum and may manifest at onset or later during the disease. ${ }^{6}$ Here, we report a rare presentation of pSS, manifesting as limbic encephalitis with reversible magnetic resonance image (MRI) findings in the bilateral medial temporal lobe.

\section{Case Report}

A 42-year-old male presented with rapid cognitive decline and memory impairment for 2 months. The history revealed, intermittent forgetfulness with gradually progressive word-finding difficulties. Despite these symptoms, he was able to dress, as well as perform activities of daily living. Two months after the first symptom, he noticed the progression of symptoms in the form of increased forgetfulness, geographic disorientation, and word-finding difficulties. His attendants complained of fluctuating consciousness with periods of profound disorientation. His cognition declined rapidly, resulting in difficulties in managing activities of daily living. There was no significant past medical or family history. He was a nonsmoker and nonalcoholic. The patient denied a history of prolonged fever, headache, vomiting, and seizures. The patient did not report any hallucinations, psychosis, or depressive symptoms. The history of high-risk sexual behavior was absent. He denied symptoms of joint pain, arthralgia, and skin rash. The patient refused exposure to drugs or toxins. On examination, the patient was conscious, cooperative, but disoriented. On the evaluation of the minimental status examination(MMSE), the score was 18 of 30.

The comprehension was intact with impairment of fluency and dysarthria. The patient's speech was found to be agrammatic, with frequent phonemic paraphasias, and impairment of repetition. The cranial nerve examination was unremarkable. Muscle bulk and tone were normal. Power was intact (5/5) on analysis. Reflexes were $2+$, and plantars were bilateral flexors. The sensory system evaluation was of 


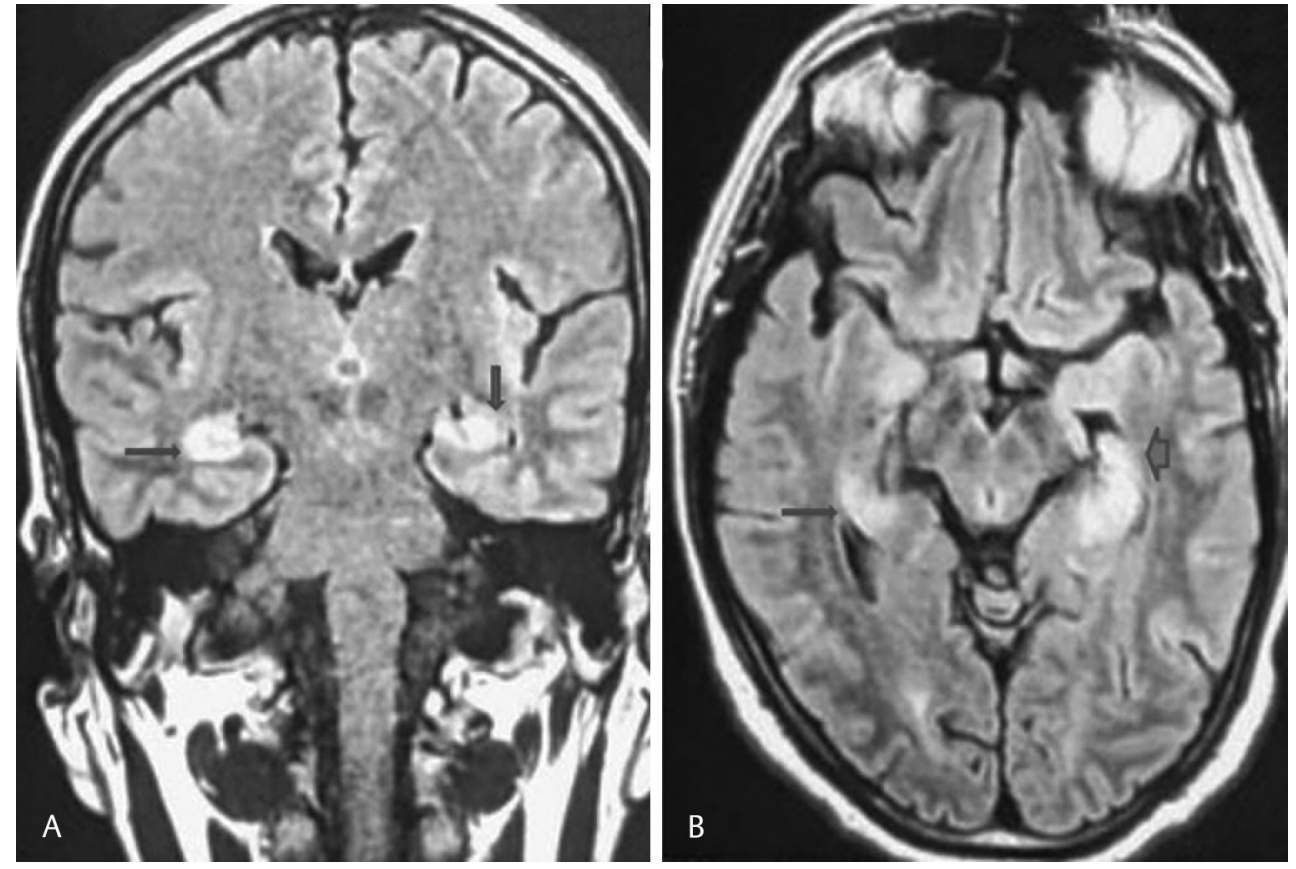

Fig.1 Fluid-attenuated inversion recovery (FLAIR) imaging, (A) coronal section and (B) axial-weighted images demonstrated hyperintense signals of mesiotemporal lobe and hippocampal region.

no note. There was no evidence of ataxia, gait imbalance, or involuntary movements. Cognitive function tests were suggestive of impaired orientation (oriented to person only), deficits in short-term memory (2/10 on word recall), verbal fluency (impaired animal naming), inability to complete trail making test in the time allotted and inability to perform complex calculations.

The hematological parameters, including total leucocyte count, platelet count, and blood cells, revealed a normal study. The renal function test, liver assessment, and thyroid study, including thyroid antibodies, were unremarkable. His serum electrolytes were within the normal range. Electroencephalography(EEG) showed predominant fast $\beta$ activity along with intermittent slow-wave discharges. Cerebrospinal fluid (CSF) examination showed mild pleocytosis (10 cells) with increased protein $(63 \mathrm{mg} / \mathrm{dL})$ and with normal sugar $(66 \mathrm{mg} / \mathrm{dL})$. CSF cytology was negative for malignant cells. CSF did not reveal oligoclonal bands, with a standard immunoglobulin (Ig)-G index. Cranial MRI revealed abnormal signals at the bilateral medial temporal lobe and hippocampal region, hyperintense on $\mathrm{T} 2$ and fluid-attenuated inversion recovery (FLAIR) sequences ( - Fig. 1). The patient was started on injection acyclovir $(10 \mathrm{mg} / \mathrm{kg} \mathrm{q} 8 \mathrm{~h}$ ) while awaiting reports of CSF virology, but the patient showed no improvement. CSF virology was negative for herpes simplex. CSF VDRL was negative. The autoimmune panel (NMDA, AMPA, GABA-B, LGI1, CASPR2 antibody), as well as paraneoplastic markers (amphiphysin, ANNA-1/Hu, ANNA-2/Ri, CV2.1, PNMA[MA2/Ta], PCA-1/Yo) were negative. The vasculitic markers, which include the antinuclear antibody(ANA) was $4+$ positive, and the extracted nuclear antigen profile showed positivity for Anti-Ro (3+). Schirmer's test was negative. The lip biopsy was suggestive of focal lymphocytic sialadenitis.

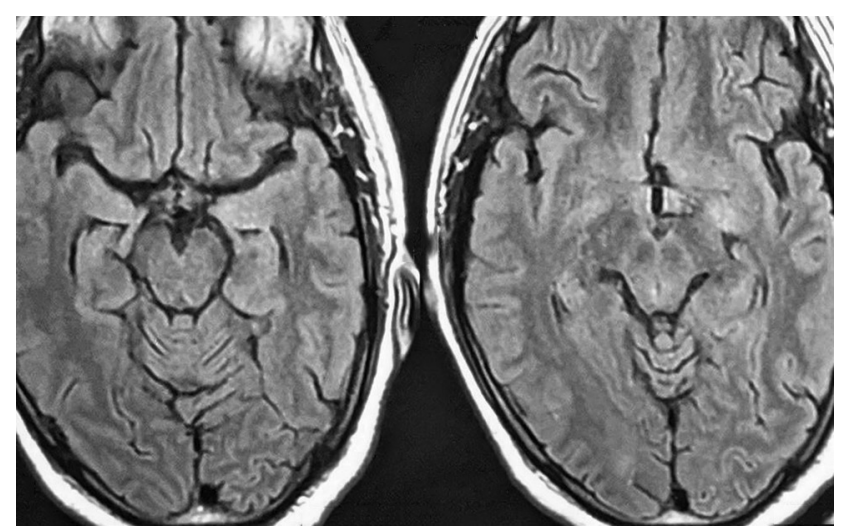

Fig. 2 Magnetic resonance imaging cranium, T2 revealed resolution of lesion.

The diagnosis of limbic encephalitis associated with pSS was established according to the 2016 American College of Rheumatology (ACR) -European League Against Rheumatism (EULAR) classification criteria. The patient was started on intravenous (IV) methylprednisolone ( $1 \mathrm{~g} \mathrm{IV} /$ day) for 5 days, followed by oral prednisolone in the dosage of $1 \mathrm{mg} / \mathrm{kg}$ ). The patient showed remarkable improvement in cognition, and in MMSE score, which was 18 of 30 on admission, became 24 of 30 after completion of MPS pulse therapy and 27 of 30 at discharge. On follow-up, the patient had complete resolution of all signs and symptoms, clinically, as well as radiologically $(\boldsymbol{- F i g}$. 2). The patient was advised to seek consultation from the rheumatology department for further treatment.

\section{Discussion}

SS is an autoimmune disorder characterized by lymphocytic and plasma cell infiltration of the exocrine glands. 
The extraglandular features include both the peripheral, as well as central nervous system involvement. PNS involvement in pSS is well studied, which manifests as cranial nerve palsies, pure or predominantly sensory polyneuropathy, sensorimotor polyneuropathy, and mononeuritis multiplex. Distal axonal sensory or sensorimotor polyneuropathy occurs most commonly and accounts for more than $50 \%$ of cases with peripheral nervous system involvement. ${ }^{7}$ On the other hand, CNS manifestations are heterogeneous and can be focal or diffuse. The diffuse pattern usually manifests as a recurrent, "subacute encephalopathy," with patients presenting with memory loss, cognitive dysfunction, and deficiencies in concentration and attention. The focal neurological manifestations include motor/sensory deficits, dysarthria, cerebellar ataxia, or seizures. Spinal cord involvement may be present and is usually manifested as transverse myelitis, neurogenic bladder, or with features of lower motor neuron disease. The CNS involvement shows a bimodal temporal pattern of distribution, presenting at onset, as well as later, during the course of illness. In approximately 25 to $60 \%$ of cases, the neurological manifestations are known to precede the diagnosis of pSS by 2 years. ${ }^{8}$ In pSS, sicca symptoms are present in only $50 \%$ of patients with neurologic involvement. Anti-Ro/SSA and anti-La/SSB antibodies are positive in only $50 \%$ of patients. ${ }^{3}$ Thus, the neurologic symptoms of SS are present in the absence of oral/ocular dryness. In such cases, the clinicians should have a high index of suspicion for pSS and consider autoantibody testing.

To the best of our knowledge, there have been only a handful of case reports associating pSS with limbic encephalitis. In the case series published by Çoban et al, the authors described three cases of pSS presenting with acute encephalopathy. The patients had features suggestive of limbic encephalitis but had either mild sicca symptoms/signs or were known cases of SS. ${ }^{9}$ Finelli and Inoa described a case of limbic encephalitis, later diagnosed as a case of SS based on Schirmer's test and pathological examination of the lip biopsy specimen. ${ }^{10}$ Unlike the previously published cases, our patient did not have any subjective or objectives signs of sicca complex, making the diagnosis more perplexing. Cognitive dysfunction and memory impairment was the sole complaints of the patient. The bilateral temporal lobe lesion was visualized in the initial neuroimaging(MRI) of our patient. Recognizing the association with limbic encephalitis (LE), patient was promptly evaluated for an infective, paraneoplastic, and autoantibody-related process. An extensive search for an underlying malignancy and alternative autoimmune, infectious, and metabolic etiologies proved unfruitful and consequently, the diagnosis was established as autoimmune LE associated with SS based on the vasculitic profile and the pathological examination of the lip biopsy. The patient was labeled as a case of pSS based on the 2016 ACR-EULAR classification criteria, with a score of $\geq 4(6 / 9)$.
Though the patient did not have objective or subjective signs of ocular and/or oral dryness, he met the inclusion criteria as the patient scored $\geq 1$ on the EULAR Sjögren's Syndrome Disease Activity Index (ESSDAI). Our patient showed excellent response to methylprednisolone pulse therapy; thereby, we can conclude that the subacute encephalopathy related to CNS involvement in SS seems to be reversible condition. ${ }^{5}$ CNS involvement in pSS is thought to be a poor prognostic marker and is related to a more aggressive pattern of progression. However, our patient showed remarkable improvement with methylprednisolone pulse therapy. Keeping in mind the association of CNS involvement with increased severity of pSS, such patients should be regularly followed-up.

\section{Conclusion}

To conclude, CNS involvement in pSS is a relatively rare complication and can manifest in a variety of ways. The CNS involvement can occur at any stage of illness and presents as a diagnostic challenge for the clinician.

\section{Funding \\ None.}

\section{Conflict of Interest}

None declared.

\section{References}

1 Fox RI. Sjögren's syndrome. Lancet 2005;366(9482) :321-331

2 Caselli RJ, Scheithauer BW, Bowles CA, et al. The treatable dementia of Sjögren's syndrome. Ann Neurol 1991; 30(1):98-101

3 Govoni M, Padovan M, Rizzo N, Trotta F. CNS involvement in primary Sjögren's syndrome: prevalence, clinical aspects, diagnostic assessment and therapeutic approach. CNS Drugs 2001;15(8):597-607

4 Moutsopoulos HM, Sarmas JH, Talal N. Is central nervous system involvement a systemic manifestation of primary Sjögren's syndrome? Rheum Dis Clin North Am 1993;19(4):909-912

5 Alexander EL. Central nervous system (CNS) manifestations of primary Sjögren's syndrome: an overview. Scand J RheumatolSuppl 1986;61:161-165

6 Alexander EL, Provost TT, Stevens MB, Alexander GE. Neurologic complications of primary Sjögren's syndrome. Medicine (Baltimore) 1982;61(4):247-257

7 Barendregt PJ, van den Bent MJ, van Raaij-van den Aarssen VJ, et al. Involvement of the peripheral nervous system in primary Sjögren's syndrome. Ann Rheum Dis 2001;60(9):876-881

8 Fauchais AL, Magy L, Vidal E. Central and peripheral neurological complications of primary Sjögren's syndrome. Presse Med 2012;41(9 Pt 2):e485-e493

9 Çoban A, Özyurt S, Meriç K, Mısırlı H, Tüzün E, Türkoğlu R. Limbic encephalitis associated with Sjögren's syndrome: report of three cases. Intern Med 2016;55(16):2285-2289

10 Finelli PF, Inoa V. Limbic encephalitis as the presenting feature of Sjögren syndrome. Neurol Clin Pract 2013;3(2): 165-167 